\title{
Cytokine and chemokine profile of the innate and adaptive immune response of schistosoma haematobium and plasmodium falciparum single and co-infected school-aged children from an endemic area of Lambaréné, Gabon
}

\author{
Ulysse Ateba-Ngoa ${ }^{1,2,3}$, Ayola Akim Adegnika ${ }^{1,2,3^{*}}$, Jeannot F Zinsou ${ }^{3}$, Roland F Kassa Kassa ${ }^{3}$, Hermelijn Smits ${ }^{1}$,
} Marguerite Massinga-Loembe ${ }^{2,3}$, Benjamin Mordmüller ${ }^{2,3}$, Peter G Kremsner ${ }^{2,3}$ and Maria Yazdanbakhsh ${ }^{1,3}$

\begin{abstract}
Background: Helminths and malaria are among the most prevalent infectious diseases in the world. They both occur in tropical area where they often affect the same populations. There are studies suggesting an effect of helminths on malariometric indices. For example, malaria attacks as well as disease severity has been shown to be influenced by a concurrent chronic helminth infection. However, there are also studies that show no effect of concurrent helminth infections on malarial outcomes. To start addressing this issue, the effect of chronic Schistosoma haematobium infection on both the innate and adaptive immune response of Plasmodium falciparum-infected subjects was assessed in an area endemic for both these infections in Gabon.

Method: Subjects infected with S. haematobium and or P. falciparum, as well as a control group with neither of these infections, were recruited. For innate immune response, heparinized blood was obtained and cultured for 24 hours with a panel of TLR ligands. For adaptive immune response, PBMC was isolated and stimulated with SEB for 72 hours. Cytokines and chemokines were measured in supernatants using a multiplex beads array immunoassay. Principal Component analysis was used to assess pattern of cytokine and chemokine responses representing the innate and adaptive components of the immune system.
\end{abstract}

Results: Overall it was observed that the presence of $P$. falciparum infection was marked by an increase in innate and adaptive immune responsiveness while $S$. haematobium infection was characterized by an increased chemokine profile, with at the same time, lower pro inflammatory markers. When the study subjects were split into single infected and co-infected groups no effect of $S$. haematobium on the immune response of $P$. falciparum infected subjects was observed, neither for the innate nor for the adaptive component of the immune response.

Conclusion: This study provides original information on the cellular immune response of S. haematobium and/or $P$. falciparum in infected subjects. It rules out an effect of S. haematobium on the cytokine profile of subjects co-infected with P. falciparum.

Keywords: Malaria, Schistosomiasis, Co-infection, Innate immune response, Adaptive immune response, Principal component analysis, Epidemiology, Lambaréné, School-aged children

\footnotetext{
* Correspondence: a.a.adegnika@medizin.uni-tuebingen.de

1 Department of Parasitology, Leiden University Medical Center, Albinusdreef 2, 2333 Leiden, ZA, The Netherlands

2Institut für Tropenmedizin, Universität Tübingen, Wilhelmstraße 27, D-72074

Tübingen, Germany

Full list of author information is available at the end of the article
} 


\section{Background}

In Plasmodium spp. infected subjects the ability to control the development of the parasite depends largely on the balance between pro and anti-inflammatory mediators of their immune response [1,2]. Acute Plasmodium falciparum infection is usually associated with an increase of INF $\gamma$ and TNF, regarded as the markers of the Th1 and pro-inflammatory response [2,3]. This proinflammatory response is thought to be needed to impede the multiplication of the parasite and favour its clearance, both in human and animal models [2-4]. While important for parasite clearance a powerful Th1 and pro-inflammatory response could also be detrimental for the host if uncontrolled, leading to tissue damage and severe disease $[5,6]$. This is supported by the importance of the anti-inflammatory network characterized by an expansion of the regulatory $\mathrm{T}$ cells [7-10], as well as by the activation of negative regulators like the CTLA4 or PD-1, transmembrane receptors, during malaria infection [11,12]. Moreover, as Th1 responses can be counteracted by Th2 cells, the presence of a strong Th2 response might also influence anti-malarial immunity.

In areas where malaria is endemic, it is the norm that Plasmodium-infected people also suffer from a concurrent helminth infection $[13,14]$. Helminths have repeatedly been shown to modulate the immune system of their host in order to survive [15]. Chronic helminthiasis is usually characterized by a marked Th2 response $[16,17]$ as well as by the induction of a regulatory network $[18,19]$ that could consequently impair the host immune response to other antigens [20]. Whether a concurrent helminth infection of the host can affect his immune response to Plasmodium spp. co-infection is still debated [21,22]. Population-based studies conducted to assess the effect of helminths on malariometric indices and on the immune response of $P$. falciparum infected subjects have so far revealed contrasting results. For example, in Senegal, Sokhna et al. observed that children with Schistosoma mansoni had an increased incidence of clinical malaria in comparison to their uninfected counterparts [23], while in Mali, Lyke and colleagues reported a protective effect of $S$. haematobium infection against malaria [24]. A similar divergent picture has also emerged when considering the cellular response of malaria and helminth co-infected subjects. For example in Senegal, Diallo et al. reported a significant increase of the plasma concentration of TNF and IFNY measured in S. haematobium and P. falciparum co-infected children in comparison to their $P$. falciparum single infected counterpart [25]. In the same studies, they also observed a significant increase of the plasma concentration of TNF, IFN $\gamma$, IL-10, TGF- $\beta$, sTNF-RI and sTNF-RII rates in co-infected subjects [25]. Similarly in Ghana, Hartgers et al. compared the cytokine response of S. haematobium subjects to uninfected ones when their whole blood were stimulated with P. falciparum infected red blood cells (iRBCs) and observed that the measured level of IL-10 was significantly higher in the infected group [26]. Inversely, in Mali Lyke et al. reported a decreased level of IL-10 in plasma from S. haematobium and malaria co-infected subjects by comparison to malaria only subjects [27].

Some reports have suggested that a concurrent helminth infection is associated with elevated cytokines in particular pro inflammatory ones compared to $P$. falciparum infected subjects $[25,26]$, while in others either no effect or even a decreased in these cytokines [27-29]. It is important to note that each of these studies assessed the immune system of infected people from a different angle, either by using different stimuli or by characterizing a different cells type. Moreover none has yet attempted to provide information on how helminths affect both the innate and the adaptive immune response of $P$. falciparum-infected subjects within the same cohort.

This study provides information on the cellular immune response of $P$. falciparum-infected subjects, with or without concurrent $S$. haematobium infection. Instead of assessing cytokines responses individually, a more global approach was taken to profile the pattern of cytokine responses in the study subjects. The study hypothesis was that a comprehensive and integrative assessment of multiple cytokines involved in the innate or the adaptive immune response of co-infected subjects would provide a better insight into the effect of $S$. haematobium on the immune response of $P$. falciparum infected subjects.

\section{Methods}

\section{Recruitment of study participants and diagnosis of parasitic infections}

This study was cross sectional and was conducted in the Bindo village located in the Moyen-Ogooué province in Gabon. The Bindo village is endemic for both S. haematobium and malaria [30]. School children from six to 16 years of age, attending the only school of the village were included. Urine and blood samples were collected at inclusion for the diagnosis of S. haematobium and P. falciparum infection as well as for immunological assays. A thorough description of the parasitological test and the immunological assays has been previously published in a study protocol article [31]. Briefly, S. haematobium infection was determined by the detection of eggs by microscopy in $10 \mathrm{ml}$ of filtrated urine. Schistosoma haematobium-uninfected subjects were those who did not show any eggs in three samples of urine collected on three consecutive days. Detection of P. falciparum infection was made by real time-PCR performed on DNA extracted from EDTA blood pellet kept frozen in $-80^{\circ} \mathrm{C}[32]$. 


\section{Immunological assays}

Peripheral blood was collected in sodium heparinized tubes for every child as described elsewhere [31]. To assess the innate immune response heparinised blood was diluted in RPMI (1:1) and cultured for 24 hours at $37^{\circ} \mathrm{C}$ with a panel of five different Toll like receptor ligands (LPS [TLR4], PAM3 [TLR1/2], CPG [TLR9] CL097 [TLR7/8]), S. haematobium eggs antigens (SEA) and a combination of LPS and SEA. Supernatant was collected after 24 hours and cytokine production was measured using the multiplex beads array immunoassay. The cytokines/chemokines quantified for this ex-vivo assay were IFN $\alpha 2$ IL-1ß, IL-6, IL-10, IL-12p70, IL-13, IFN $\gamma$, MCP-1, MIP-1 $\alpha$, MIP-1ß, TNF and IP-10.

In order to assess the adaptive immune response, PBMC were isolated by density gradient centrifugation on Ficoll as already described [31]. PBMCs were cultured for 72 hours at $37^{\circ} \mathrm{C}$ with SEB and supernatant was collected for the measurement of a panel of 11 different cytokines (TNF, IFNy, IL-2, IL-4, IL-5, IL-13, IL17A, IL-17 F, IL-22, IL-10, and IL-21) by a multiplex beads array immunoassay.

\section{Statistics}

Chi square and fisher's exact test were used to compare categorical variables. Not normally distributed quantitative variables were transformed either by Log10 or BoxCox transformation. Student $t$-test and ANOVA were performed when data met the assumption of normality. Otherwise the non-parametric Mann Whitney and Kruskal Wallis tests were used. Correlation between two continuous variables was assessed using the spearman rho test.

Principal component analysis (PCA) was carried out on the cytokine variables in order to summarize them. Of note PCA is a mathematical technique that allows reducing the dimension of large dataset by identifying new summary variables also called principal component (PC). Each principal component is made of a set of original variables that share a certain level of correlation. All the analysis was performed on medium subtracted data. Negative values were set to zero. Cytokine from the innate [obtained after whole blood stimulation] and the adaptive panels [obtained after PBMC stimulation] were analyzed independently by PCA. As mentioned above the innate panel consisted of 12 cytokines measured after stimulation of cells by six different conditions. Before performing the PCA an average cytokine response were calculated for each subject by taking the mean of the cytokine level obtained with the six different stimuli. This step only concerned the innate panel and was not needed for cytokine of the adaptive panel since the cytokine response to one stimulus was only assessed. All variables were transformed either using a $\log 10$ or a
Box-Cox transformation to reduce skewness prior to PCA. Principal components identified were considered for further analysis if their eigenvalues were above one. No rotation was applied. Individuals PC scores were obtained for each subject and used for comparison between groups. All the statistical tests were computed using $\mathrm{R}$ version 3.0.1. The $R$ packages ggplot2 version 0.9.3.1 and FactoMineR version 1.25 were used for making graphs and performing the PCA respectively. Statistical significance was set for $\mathrm{p}$ value below 0.05 .

\section{Ethics}

The study was approved by the "Comité d'éthique Régional de Lambaréné" (CERIL). Informed consent was obtained from parents or legal guardians of each of the children included in the study. Appropriate treatment was given to children found with $P$. falciparum or $S$. haematobium infection as per the local guidelines.

\section{Results}

\section{Characteristics of the study subjects}

The recruitment of the study participants took place in May 2011. Overall 125 subjects aged from six to 16 years were included. Among them 63 (50.4\%) were infected with S. haematobium while 66 (53\%) carried P. falciparum in their blood as determined by PCR. When considering coinfection four different groups were compared as shown in Table 1. Children with $P$. falciparum single infection were younger and had a lower haemoglobin level. No other significant differences were found between the different groups.

\section{Innate immune responses}

Cytokine and chemokines were measured following the stimulation of whole blood with a panel of TLR ligands for 24 hours. In Figure 1a, the levels of measureable chemokines/cytokines are shown. The chemokines/cytokines showed a certain degree of correlation (Figure 2a) that prompted us to perform a principal component analysis. The PCA identified two PCs (respectively named innate PC1 (iPC1) and $\mathrm{iPC} 2$ ) that summarized the 12 measured chemokines/cytokines. These PCs are described in Table 2. Briefly the iPC1 comprised of almost all chemokines/cytokines included in the PC analysis and, therefore, was interpreted as reflecting the general responsiveness. The iPC2 was best characterized by four chemokines/ cytokines that clustered into two groups; the MCP1-MCAF/ MIP-1 $\beta$, which were positively loaded and the INF $\gamma /$ TNF, which were negatively loaded in the PCs. In other words an increase of $\mathrm{PC} 2$ would represent an increase of MCP1-MCAF/MIP-1 $\alpha$ and a simultaneous decrease of INF $\gamma /$ TNF.

Neither age nor gender affected iPC1 or iPC2. In a univariate analysis, $\mathrm{PCC} 1$ was higher in P. falciparum 
Table 1 Characteristics of the study subjects divided by infection status

\begin{tabular}{|c|c|c|c|c|c|}
\hline & S.h-/ P.f- & S.h +/ P.f- & S.h -/ P.f+ & S.h +/ P.f+ & $\mathrm{p}$-value \\
\hline Number of subjects (\%) & $28(22 \%)$ & $31(25 \%)$ & $34(27 \%)$ & $32(26 \%)$ & - \\
\hline Gender: M/F & $14 / 14$ & $13 / 18$ & $19 / 15$ & $18 / 14$ & 0.63 \\
\hline Age in years: Median (IQR) & $11(3.25)$ & $11(5)$ & $9.5(4)$ & 13(3) & 0.02 \\
\hline Weight in kgs: Median(IQR) & $31.5(12.25)$ & $35(15)$ & $30(9.8)$ & $36(18.25)$ & 0.05 \\
\hline Haemoglobin in g/dl: Median (IQR) & $11.9(0.8)$ & $12.25(0.95)$ & $11.1(1.08)$ & 11.8(1.6) & 0.003 \\
\hline Number of subjects living in the village for more than 5 years (\%) & $17(61 \%)$ & $21(75 \%)$ & $15(44 \%)$ & 19(61\%) & 0.10 \\
\hline Number of filaria infected subjects (\%) & $2(7 \%)$ & $4(13 \%)$ & $2(6 \%)$ & $2(6 \%)$ & 0.67 \\
\hline Number of subjects previously treated for S. haematobium: (\%) & $9(32 \%)$ & $21(41 \%)$ & $7(21 \%)$ & $14(45 \%)$ & 0.16 \\
\hline S. haematobium eggs count per $10 \mathrm{ml}$ : Median (IQR) & 0 & 15(49.5) & 0 & $52.5(88.5)$ & $0.26^{\#}$ \\
\hline P. falciparum CT value Median (IQR) & 0 & 0 & $28.9(7.3)$ & $30.7(8.6)$ & $0.25^{\#}$ \\
\hline
\end{tabular}

S.h-/ P.f- : Subjects not infected by either S. haematobium or P. falciparum. S.h+/ P.f-: Subjects with single S. haematobium infection. S.h-/ P.f+: Subjects with single $P$. falciparum infection. S.h+/ P.f+: S. haematobium and P. falciparum co-infected subjects. M: male and F: female. CT value represents the value of the cycle threshold.

\#p-value computed to compare infected subjects only.

infected subjects in comparison to subjects with no $P$. falciparum infection (median level of the iPC1 scores: 0.133 in infected $v s 0.003$ in uninfected subjects, $\mathrm{p}=0.019$, Figure 3a), whereas $S$. haematobium infection was associated with an increased iPC2 (0.6 in infected vs 0.04 in uninfected, $\mathrm{p}=0.016$, Figure $3 \mathrm{~b}$ ). This indicates that during $P$. falciparum infection there is an enhancement of responses to innate stimuli in general while $S$. haematobium infection appears to lead to a selective increase in the release of macrophage-released chemokines, and at the same time to a decrease of pro-inflammatory cytokines in response to TLR stimuli.

To further assess immune response in single and coinfected subjects with particular emphasis on the question
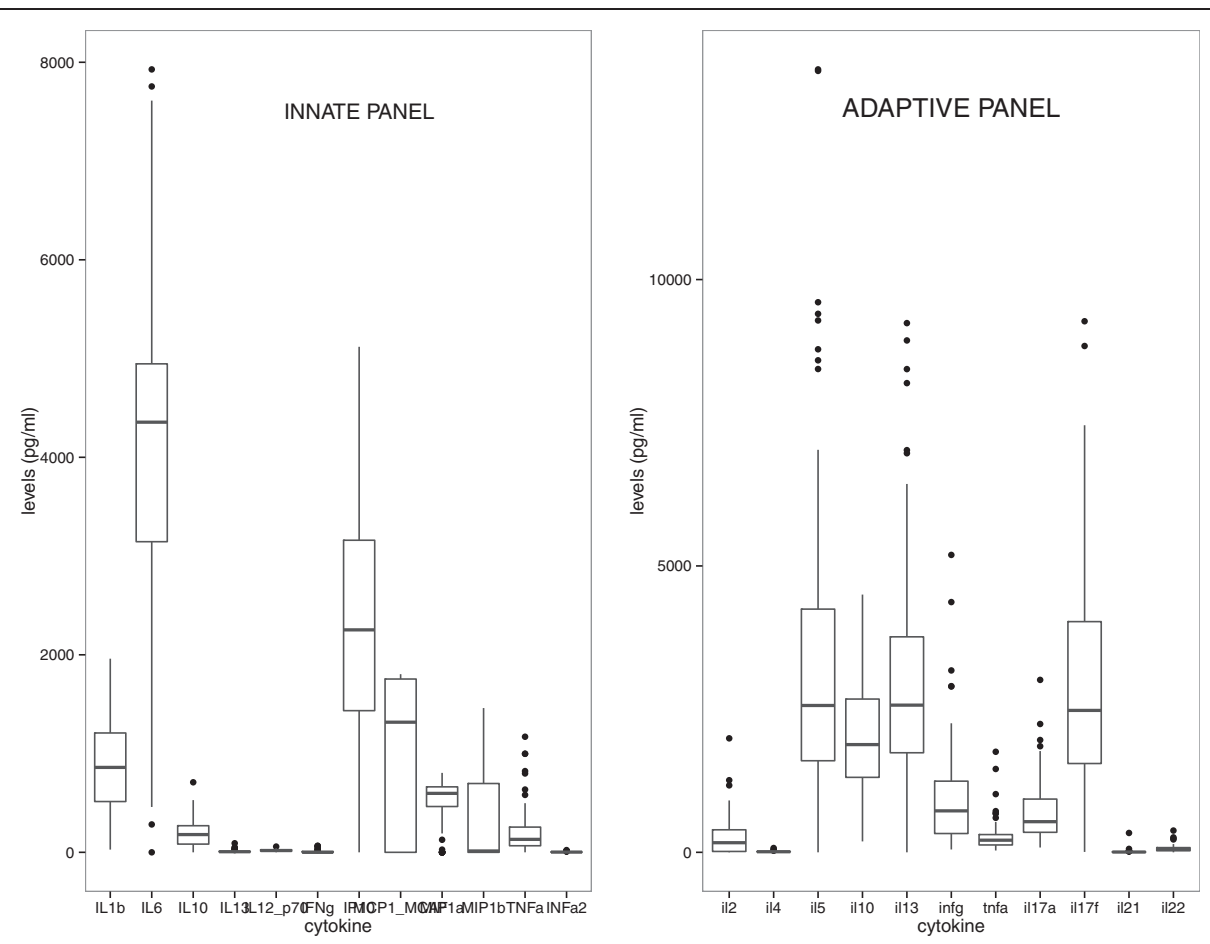

Figure 1 Levels of the cytokines measured for the innate (left) and the adaptive (right) panels. For the innate panel the mean response was calculated per cytokine for the 6 different antigens that were used in the whole blood assay. This step was not needed for cytokine pertaining to the adaptive panel since only the cytokine response after SEB stimulation was assessed. Boxes represent the magnitude of the overall response of the study subjects per each cytokine. Whiskers represent minimal and maximal concentrations and dots are indicative of subjects with outlier values. 


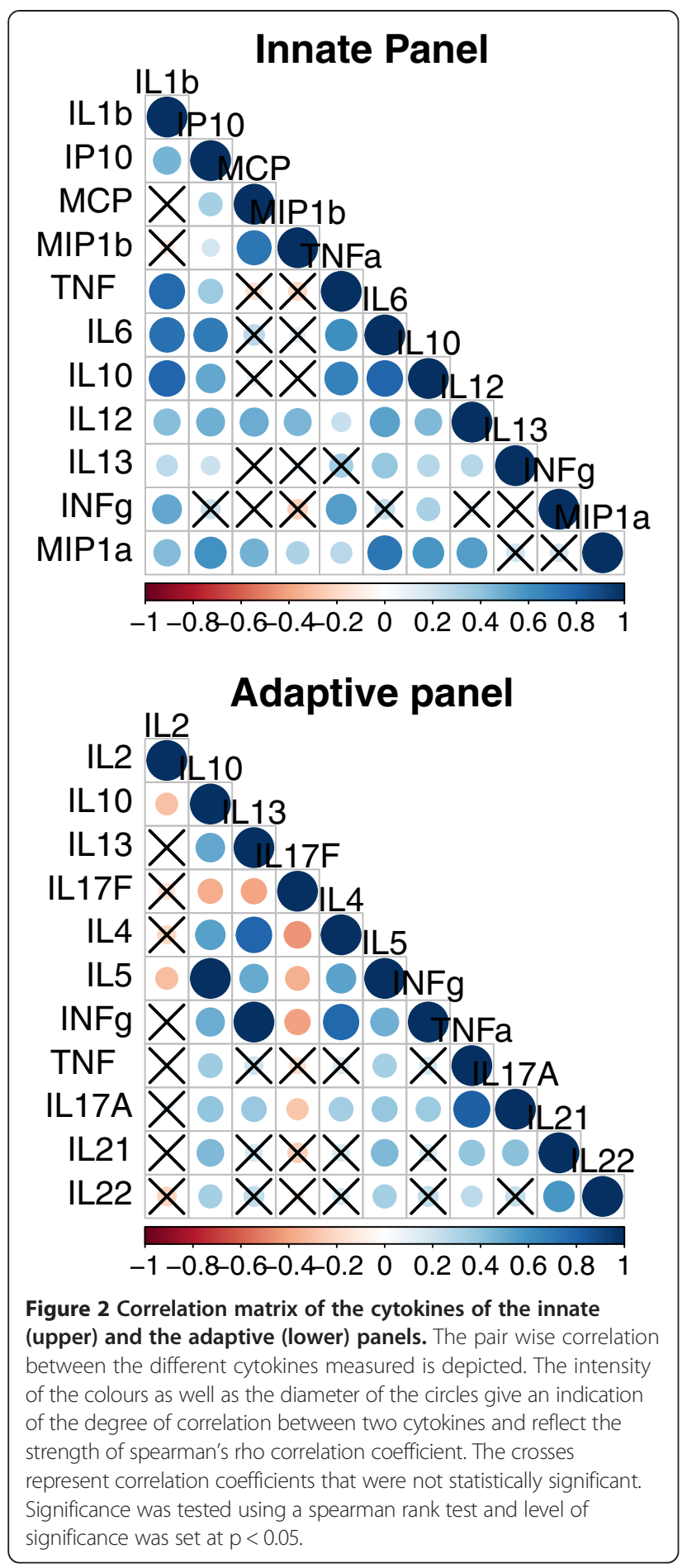

whether S. haematobium affects response associated with $P$. falciparum, the study population was divided into four groups of uninfected, infected with $P$. falciparum only, $S$. haematobium only and infected with both. What was observed was that iPC1 was higher in those with P. falciparum infection, and statistically significantly so in those co-infected with S. haematobium (Table 3). These data indicate that $P$. falciparum effect on the immune system is not influenced by concurrent $S$. haematobium infection.

\section{Adaptive immune responses}

As TLRs stimulate the innate immune system in general, to assess the general response of the adaptive immune system the PBMC were stimulated with SEB which is a superantigen capable of triggering a polyclonal $\mathrm{T}$ cells activation, part of the general adaptive immune responsiveness. The cytokines measured are shown in Figure $1 \mathrm{~b}$ and the extent of their correlation in Figure 2b. To profile the cytokine response, a PCA was performed. As shown in Table 2, three different PCs (adaptive PC1 (aPC1), aPC2 and aPC3) were identify. Based on the type of cytokines that contributed to the $\mathrm{PC}$, they were interpreted as follows: aPC1 represented the general immune responsiveness; aPC2 the Th2/Th17; and aPC3 Th1/ Th17 response.

There were no differences between males and females for the aPC1, aPC2, and aPC3 (data not shown). A strong correlation between $\mathrm{aPC} 1$ and the age of the study participants (rho $=0.3, \mathrm{p}<0.008$ ) was observed but no effect of age was seen on aPC2 (rho $=0.1, \mathrm{p}=0.3$ ) or aPC3 (rho $=-0.1, \mathrm{p}=0.4)$. As shown in Figure 4a, a trend toward an increase of the aPC1 was seen with $P$. falciparum infection but no effect was observed on the aPC2 or aPC3. Moreover none of the components, aPC1, aPC2 or aPC3 was affected by S. haematobium infection (Figure 4b). Finally, no statically significant differences between groups were detected when $P$. falciparum and $S$. haematobium co-infected subjects were compared with those with single or no infection (Table 3).

\section{Discussion}

The main objective of this study was to determine whether chronic S. haematobium infection was able to affect the cellular immune response of $P$. falciparum infected subjects. By measuring the cytokine production after in-vitro stimulation, the innate and adaptive immune responses of the study subjects were profiled. Here, rather than assessing single cytokines, the pattern of cytokine responses of the study subjects using a PCA was evaluated. PCA is a mathematical tool widely used in the field of biology. It has the advantage of summarizing highly correlated variables in new latent and synthetic variables called principal components that can unveil new pattern of responses [33,34]. Two PCs (iPCs) that summarize the innate cytokine responses of the study participants were identify as well as 3 PCs (aPCs) for the adaptive cytokine responses. The interpretation of these different PCs shows that cytokines are released with a certain degree of correlation. This is supported by the fact that none of the PCs identified was made of only one cytokine and at least two cytokines were represented in every PC. Moreover, 
Table 2 Description of the different principal components identified for the innate panel (iPC1 and iPC2) and the adaptive panel (aPC1, aPC2 and aPC3)

\begin{tabular}{|c|c|c|c|c|c|c|c|c|c|c|}
\hline \multirow{2}{*}{$\begin{array}{l}\text { Cytokines/ } \\
\text { Chemokines }\end{array}$} & \multicolumn{2}{|l|}{ iPC1 } & \multicolumn{2}{|l|}{ iPC2 } & \multicolumn{2}{|l|}{ aPC1 } & \multicolumn{2}{|l|}{ aPC2 } & \multicolumn{2}{|l|}{$\mathrm{aPC} 3$} \\
\hline & Score & Contribution & Score & Contribution & Score & Contribution & Score & Contribution & Score & Contribution \\
\hline IL1b & 0.4 & 14 & -0.2 & 6 & - & - & - & - & - & - \\
\hline IP10 & 0.3 & 11 & - & - & - & - & - & - & - & - \\
\hline MCP1-MCAF & 0.2 & 2 & 0.5 & 27 & - & - & - & - & - & - \\
\hline MIP1a & 0.3 & 12 & 0.2 & 5 & - & - & - & - & - & - \\
\hline MIP1b & 0.1 & 1 & 0.5 & 29 & - & - & - & - & - & - \\
\hline TNF & 0.3 & 10 & -0.4 & 13 & - & - & - & - & - & - \\
\hline IL6 & 0.4 & 17 & - & - & - & - & - & - & - & - \\
\hline IL10 & 0.4 & 14 & -0.1 & 2 & - & - & - & - & - & - \\
\hline IL12 & 0.3 & 9 & 0.3 & 8 & - & - & - & - & - & - \\
\hline IL13 & 0.2 & 4 & - & - & - & - & - & - & - & - \\
\hline INFY & 0.2 & 4 & -0.3 & 10 & - & - & - & - & - & - \\
\hline IL5 & - & - & - & - & 0.34 & 11.7 & 0.55 & 29.8 & -0.13 & 1.7 \\
\hline IL10 & - & - & - & - & 0.39 & 15.1 & 0.21 & 4.6 & -0.03 & 0.1 \\
\hline IL13 & - & - & - & - & 0.35 & 12.6 & 0.49 & 23.7 & -0.18 & 3.4 \\
\hline INFY & - & - & - & - & 0.37 & 13.7 & -0.24 & 5.6 & 0.56 & 31 \\
\hline TNF & - & - & - & - & 0.42 & 17.6 & -0.09 & 0.9 & 0.49 & 24.4 \\
\hline IL17A & - & - & - & - & 0.36 & 13.2 & -0.31 & 9.8 & -0.13 & 1.6 \\
\hline IL21 & - & - & - & - & -0.31 & 9.3 & 0.32 & 10.8 & 0.42 & 17.6 \\
\hline IL22 & - & - & - & - & -0.26 & 6.9 & 0.38 & 14.5 & 0.45 & 20.23 \\
\hline
\end{tabular}

it was noticed that within the same PC cytokines were either negatively or positively correlated. For example in the iPC2, Th1 type cytokines (IFN $\gamma$ and TNF) were negatively correlated with cytokines released by macrophages (MCP1-MCAF and MIP1 $\beta$ ) implying an antagonistic effect that may need further investigations.

In a number of studies it has been shown that $S$. haematobium infection can influence the innate immune response of the human host. For instance in population based studies, schistosomiasis has been linked with functional impairment of human myeloid dendritic cells [35] and their response to TLR ligands [36-38]. Schistosoma haematobium excretory-secretory products can prime dendritic cells to shape the adaptive response toward a Th2 phenotype [38,39]. While this immune profile is thought to limit the damage caused by schistosomes in the human host, it could alter the host immune response to a concurrent $P$. falciparum co-infection. What was observed in this study is that $P$. falciparum infection was marked by an increase of the iPC1 and aPC1, which represented the innate and adaptive general immune responsiveness. Interestingly, this was not the case for $S$. haematobium infection, which was associated with an increased level of chemokines (MCP1-MCAF and MIP1b) and the decrease of pro-inflammatory cytokines, namely INF $\gamma$ and TNF. This indicates that the immune system responds differently to $P$. falciparum and to $S$. haematobium infection. In $P$. falciparum-infected subjects the increase of the $\mathrm{PPC} 1$ and aPC1 component is in line with the immune profile seen in asymptomatic $P$. falciparum infected subjects [40]. This is also in line with the literature indicating that in subjects chronically infected with $S$. haematobium there is a down modulation of the pro inflammatory response that is thought to allow the survival of the parasites $[18,19]$. These observations regarding $S$. haematobium are in line with results of two independent studies that assessed the innate immune response of schistosome-infected subjects. In the first study, Turner et al. observed that upon stimulation of whole blood with schistosome excretory/secretory products, S. haematobium infected subjects had an enhanced production of IL-10, an anti inflammatory cytokine, whereas the level of the pro-inflammatory cytokine TNF was not different from the uninfected subjects [41]. In the second study, Van der Kleij et al. observed that $S$. haematobium infection was associated with a significant decrease in responsiveness to LPS irrespective of pro or anti inflammatory cytokines [37]. However, a study by Meurs et al. reported that PBMC of S. haematobium infected subjects produced significantly more TNF after stimulation with Pam3 a TLR2/1 ligand in comparison to their S. haematobium uninfected counterparts [36]. These 


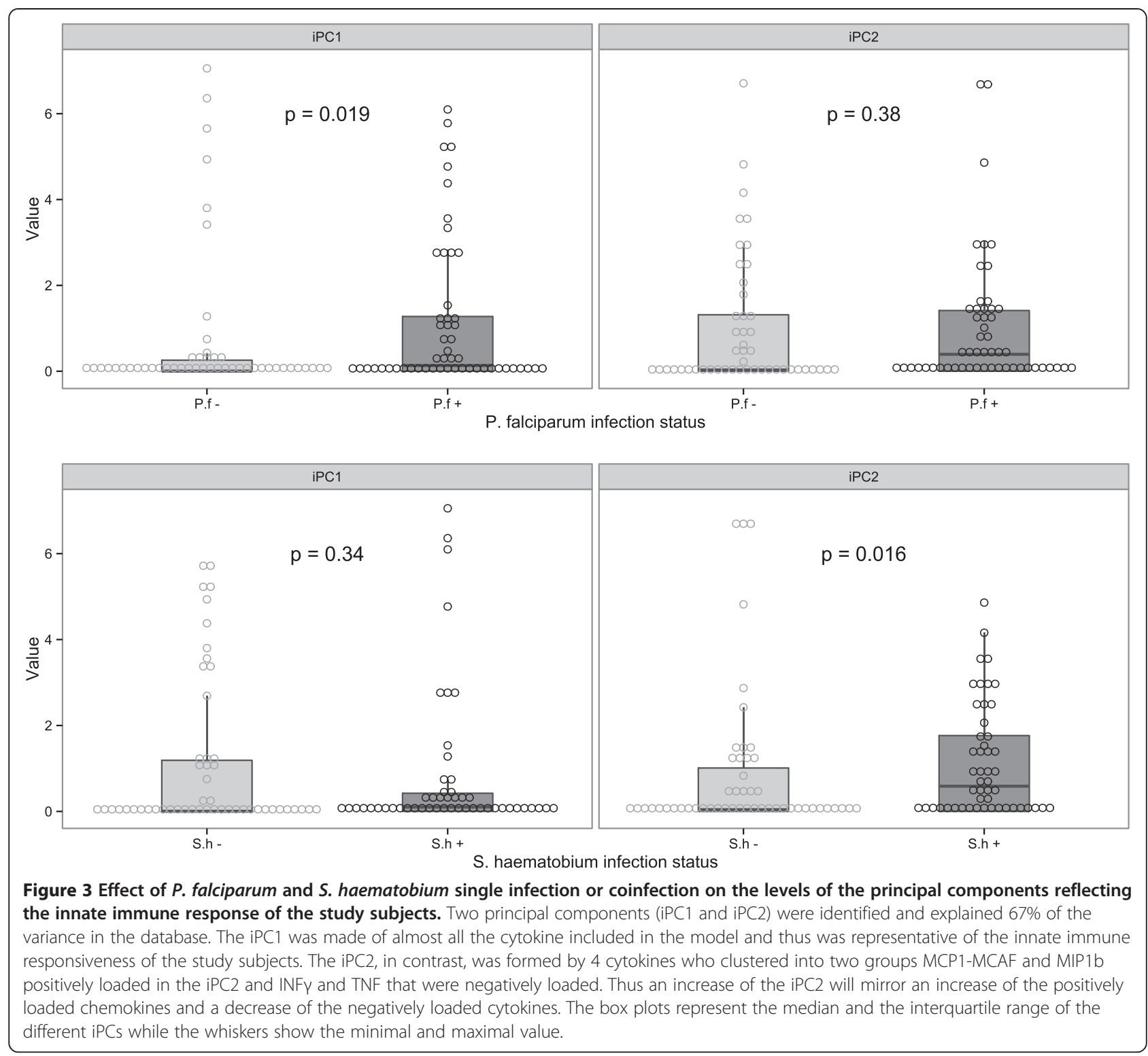

Table 3 Effect of $P$. falciparum (P.f.) and S. haematobium (S.h) co-infection on the different principal components identified from the innate (iPC) and the adaptive (aPC) immune response

\begin{tabular}{lllllc}
\hline & $\begin{array}{l}\text { S.h-/P.f- } \\
\text { Median (IQR) }\end{array}$ & $\begin{array}{l}\text { S.h }+ \text { / P.f }- \\
\text { Median (IQR) }\end{array}$ & $\begin{array}{l}\text { S.h }-/ \text { P.f }+ \\
\text { Median (IQR) }\end{array}$ & $\begin{array}{l}\text { S.h +/ P.f }+ \\
\text { Median (IQR) }\end{array}$ \\
\hline iPC1 & $0(0-0.03)$ & $0.05(0-0.31)$ & $0.24(0-1.28)$ & $0.13(0.005-0.94)$ \\
iPC2 & $0(0-0.31)$ & $0.73(0-2.37)$ & $0.12(0-1.22)$ & $0.53(0.005-1.48)$ \\
aPC1 & $0.26(0.11-0.89)$ & $0.38(0.05-1.25)$ & $0.36(0.11-1.4)$ & $1(0.18-3.31)$ & $0.047^{\# \#}$ \\
aPC2 & $0.53(0.18-0.84)$ & $0.66(0.2-1.26)$ & $1.27(0.4-1.7)$ & $0.75(0.2-1.6)$ & 0.22 \\
aPC3 & $0.6(0.25-1.33)$ & $0.47(0.1-1.6)$ & $1.34(0.24-3.019)$ & $0.25(0.05-1.1)$ & 0.42 \\
\hline
\end{tabular}

S.h-/ P.f- : Subjects not infected by either S. haematobium or P. falciparum. S.h+/ P.f-: Subjects with single S. haematobium infection. S.h-/ P.f+: Subjects with single $P$. falciparum infection. S.h+/ P.f+: S. haematobium and $P$. falciparum co-infected subjects. M: male and F: female. CT value represents the value of the cycle threshold. "Two by two comparison of the groups are shown and show significant difference between S.h-/P.f- vs S.h $+/$ P.f $+(p=0.04)$. ${ }^{\# \#}$ Two by two comparison show significant difference between S.h-/P.f- vs S.h $+/$ P.f - and between $S . h-/ P . f-$ vs $S . h+/ P . f+(p=0.03$ and 0.02 respectively). 


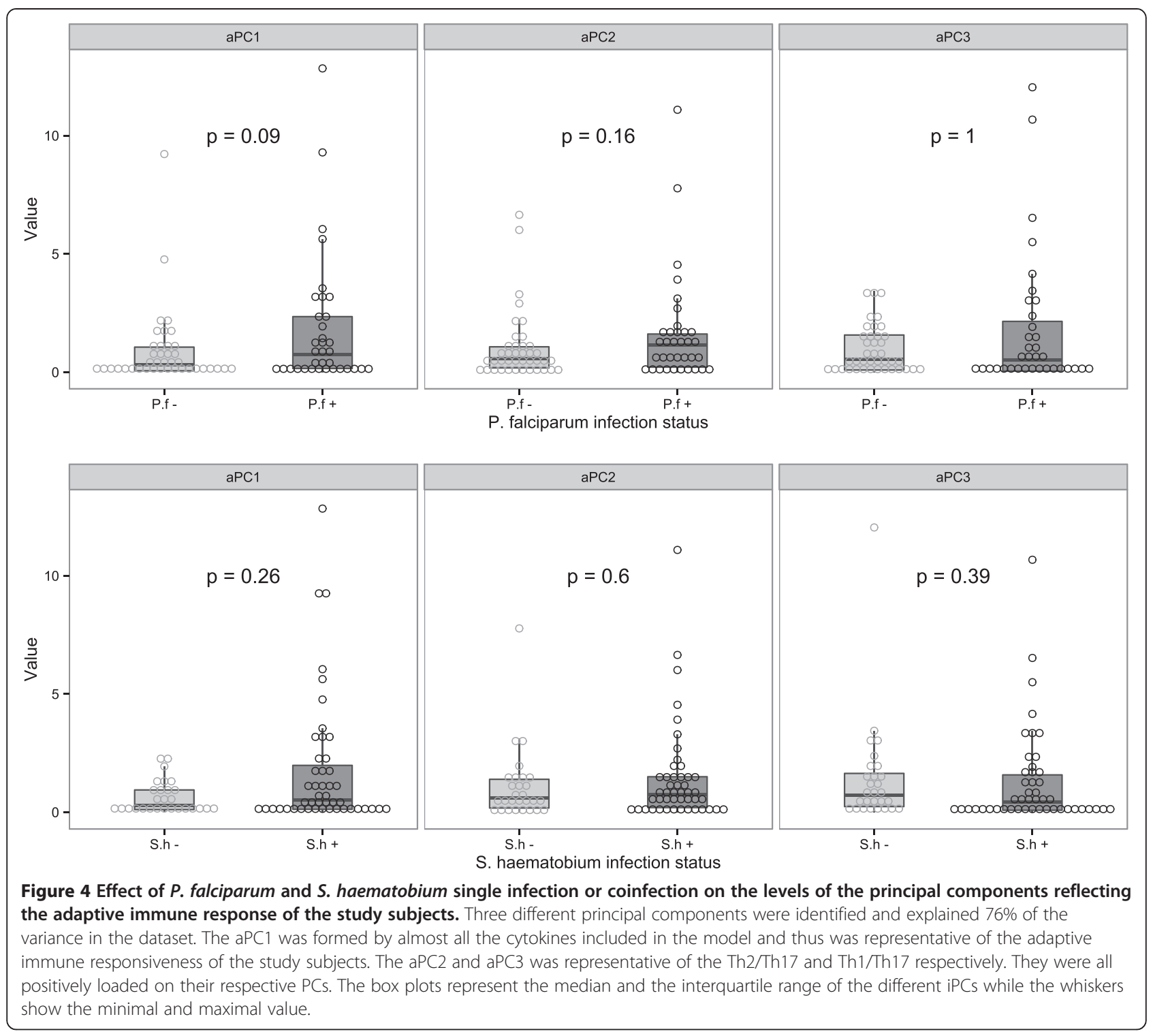

differences are difficult to reconcile but the culture methods [whole blood versus PBMC], seasonal fluctuation in immune responses, or other factors such as different environments or co infections [42,43], need to be taken into consideration when comparing studies.

This study did not observe an effect of $S$. haematobium on the innate and adaptive cytokine profile of $P$. falciparum infected subjects. The current body of evidence on helminth and malaria co-infection and its effect on the host immune response has so far given contrasting results. For example a cross sectional study showed no impact of light intensity Ascaris infection on the immune response of malaria infected subjects [44]. In a study conducted in Mali, S. haematobium infected and uninfected subjects were followed up until the time to the first malaria episode and serum cytokines were measured at the time of inclusion and at the time when study subjects became infected with $P$. falciparum [27]. At baseline the level of IL-4, IL-6, IL-10 and IFN $\gamma$ cytokines were all higher in subjects infected with S. haematobium by comparison to uninfected subjects. However, when these participants developed an acute episode of malaria, IL-6 and IL-10 cytokines increased considerably in all groups, but to a higher extent in subjects who were free of schistosome infection [27], which would suggest that $S$. haematobium impedes the cytokine storm. It has to also be noted that, looking at the results differently, which is that at the time of malaria infection, the baseline differences in IL-6 and IL-10 in the $S$. haematobium infected and uninfected subjects, fell short of statistical significance, one might conclude that there is no difference between subjects with single malaria versus those who were coinfected. In contrast, in a study in Senegal, where P. falciparum 
infected participants were compared to $S$. haematobium and P. falciparum co-infected subjects; Diallo et al. reported that the plasma concentration of IL-10, TGF $\beta$, INFY (but not INF $\alpha$ ) was higher in co-infected subjects than in those with single infection. The same authors, when examining in vitro production of cytokines by mononuclear cells stimulated with $P$. falciparum schizont extracts and MSP1-19 antigens reported an increase of IL-10 and INF $\gamma$ but not TGF $\beta$, IL-12 or IL-13 in subjects with $P$. falciparum infection compared with subjects co-infected with $P$. falciparum and S. haematobium [45]. Finally, a study conducted by Hartgers et al. in Ghana showed higher response to malaria antigens in terms of IL-10 but not INF $\gamma$, IL-6, TNF in helminth infected subjects in comparison to those free of helminth infection [26]. It is important to emphasize that in the study of Hartgers et al., the response to malaria antigens was compared between $S$. haematobium-infected and uninfected subjects and, therefore, malaria infection was artificially mimicked by the use of antigens from P. falciparum. Regarding, the Senegal studies, the P. falciparum singly infected individuals originated from a village where S. haematobium infection was never reported before, whereas co-infected subjects were from an entirely different village endemic for both $S$. haematobium and $P$. falciparum. Therefore, it is possible that the differences reported, mirror the exposure to different environmental factors rather than to S. haematobium. This is supported by the work by Smolen and colleagues who compared the immune response of children across four different continents. Using a standardized procedure they observed considerable heterogeneity in the cytokine responses in the different geographical areas [42].

One obvious limitation of the present study is that it is cross sectional and one could argue that it does not provide information on history of past helminth infections that are capable of imprinting the host immune system. For example, the innate immune system has been shown to be able to keep a "memory" of early exposure to PAMPs through a process called "trained immunity" which is not addressed in this study [46]. Additional limitation concerns the sample size of the study that may not be sufficient to detect an effect of helminths on P. falciparum modulated immune responses. However, this study was carried out in a relatively small community where it was possible to enroll all the school-aged children willing to participate and fulfilling the inclusion criteria. Despite these limitations the present study provides original information on the cellular immune response of S. haematobium and/or P. falciparum infected subjects. It showed that $P$. falciparum, but not $S$. haematobium, infection was associated with an increase of the immune responsiveness of the study subjects but it did not evidenced an effect of $S$. haematobium on the immune response that were measured in the $P$. falciparuminfected participants.

\section{Conclusions}

This study assessed the effect of S. haematobium on the pattern of cytokine responses elicited in subjects concurrently infected with $P$. falciparum. It shows that $P$. falciparum infection is associated with an increased immune responsiveness which is not affected by $S$. haematobium co-infection.

\section{Competing interests}

The authors declare that they have no competing interests.

\section{Authors' contributions}

UAN, JFZ, RFKK carried on the study on the field. They were responsible of the screening and the enrolment of the study participants. UAN carried on the different immunological assays, performed the statistical analysis and wrote the first draft. AAA, MML and BM advise on the epidemiological aspect of the study. HS advise on the immunological aspect of the study. PGK, MY and AAA designed and coordinated the study. All authors participated in the manuscript preparation, read and approved the final version of the manuscript.

\section{Acknowledgements}

This work was supported by: the European Union funded project : An AfricanEuropean Research Initiative (IDEA)" (HEALTH-F3-2009-241642); the EDCTP Project code TA.11.40200.025" and the Deutsche Forschungsgemeinschaft-funded project Deutsch-Afrikanische Kooperationsprojekte in der Infektiologie (DFG-Projekt KR 1150/6-1. We acknowledge support by Deutsche Forschungsgemeinschaft and Open Access Publishing Fund of Tuebingen University. The funders had no role in study design, data collection and analysis, decision to publish, or preparation of the manuscript.

\section{Author details}

${ }^{1}$ Department of Parasitology, Leiden University Medical Center, Albinusdreef 2, 2333 Leiden, ZA, The Netherlands. ${ }^{2}$ Institut für Tropenmedizin, Universität Tübingen, Wilhelmstraße 27, D-72074 Tübingen, Germany. ${ }^{3}$ Centre de Recherches Médicales de Lambaréné, BP: 118, Lambaréné, Gabon.

Received: 20 August 2014 Accepted: 9 February 2015

Published online: 25 February 2015

\section{References}

1. Clark IA, Alleva LM, Budd AC, Cowden WB. Understanding the role of inflammatory cytokines in malaria and related diseases. Travel Med Infect Dis. 2008;6:67-81.

2. Winkler S, Willheim M, Baier K, Schmid D, Aichelburg A, Graninger W, et al. Reciprocal regulation of Th1- and Th2-cytokine-producing T cells during clearance of parasitemia in Plasmodium falciparum malaria. Infect Immun. 1998;66:6040-4.

3. Clark IA, Budd AC, Alleva LM, Cowden WB. Human malarial disease: a consequence of inflammatory cytokine release. Malar J. 2006;5:85.

4. Perlaza B-L, Sauzet J-P, Brahimi K, BenMohamed L, Druilhe P. Interferon- $\gamma$, a valuable surrogate marker of Plasmodium falciparum pre-erythrocytic stages protective immunity. Malar J. 2011;10:27.

5. Palomo J, Fauconnier M, Coquard L, Gilles M, Meme S, Szeremeta F, et al. Type I interferons contribute to experimental cerebral malaria development in response to sporozoite or blood-stage Plasmodium berghei ANKA. Eur J Immunol. 2013;43:2683-95.

6. Villegas-Mendez A, Greig R, Shaw TN, de Souza JB, Gwyer Findlay E, Stumhofer JS, et al. IFN- $\gamma$-producing CD4+ T cells promote experimental cerebral malaria by modulating CD8+ T cell accumulation within the brain J Immunol. 2012;189:968-79.

7. Nie CQ, Bernard NJ, Schofield L, Hansen DS. CD4+ CD25+ regulatory T cells suppress CD4+ T-cell function and inhibit the development of Plasmodium berghei-specific $\mathrm{TH} 1$ responses involved in cerebral malaria pathogenesis. Infect Immun. 2007;75:2275-82. 
8. Haque A, Best SE, Amante FH, Mustafah S, Desbarrieres L, de Labastida F, et al. CD4+ natural regulatory T cells prevent experimental cerebral malaria via CTLA-4 when expanded in vivo. PLoS Pathog. 2010;6:e1001221.

9. Finney OC, Nwakanma D, Conway DJ, Walther M, Riley EM. Homeostatic regulation of $\mathrm{T}$ effector to Treg ratios in an area of seasonal malaria transmission. Eur J Immunol. 2009;39:1288-300.

10. Walther $M$, Tongren JE, Andrews $L$, Korbel $D$, King $E$, Fletcher $H$, et al. Upregulation of TGF-beta, FOXP3, and CD4 + CD25+ regulatory T cells correlates with more rapid parasite growth in human malaria infection. Immunity. 2005:23:287-96.

11. Hafalla JCR, Claser C, Couper KN, Grau GE, Renia L, de Souza JB, et al. The CTLA-4 and PD-1/PD-L1 inhibitory pathways independently regulate host resistance to Plasmodium-induced acute immune pathology. PLoS Pathog. 2012;8:e1002504

12. Butler NS, Moebius J, Pewe LL, Traore B, Doumbo OK, Tygrett LT, et al. Therapeutic blockade of PD-L1 and LAG-3 rapidly clears established bloodstage Plasmodium infection. Nat Immunol. 2012;13:188-95.

13. Brooker SJ, Pullan RL, Gitonga CW, Ashton RA, Kolaczinski JH, Kabatereine $\mathrm{NB}$, et al. Plasmodium-helminth coinfection and its sources of heterogeneity across East Africa. J Infect Dis. 2012;205:841-52.

14. Adegnika AA, Kremsner PG. Epidemiology of malaria and helminth interaction: a review from 2001 to 2011. Curr Opin HIV AIDS. 2012;7:221-4.

15. Hartgers FC, Yazdanbakhsh M. Co-infection of helminths and malaria: modulation of the immune responses to malaria. Parasite Immunol. 2006:28:497-506.

16. Grzych JM, Pearce E, Cheever A, Caulada ZA, Caspar P, Heiny S, et al. Egg deposition is the major stimulus for the production of Th2 cytokines in murine schistosomiasis mansoni. J Immunol. 1991;146:1322-7.

17. De Oliveira Fraga LA, Torrero MN, Tocheva AS, Mitre E, Davies SJ. Induction of type 2 responses by schistosome worms during prepatent infection. J Infect Dis. 2010;201:464-72

18. Wammes LJ, Hamid F, Wiria AE, Wibowo H, Sartono E, Maizels RM, et al. Regulatory $T$ cells in human lymphatic filariasis: stronger functional activity in microfilaremics. PLoS Negl Trop Dis. 2012;6:e1655.

19. Nausch N, Midzi N, Mduluza T, Maizels RM, Mutapi F. Regulatory and activated T cells in human Schistosoma haematobium infections. PLoS One. 2011;6:e16860

20. Sabin EA, Araujo MI, Carvalho EM, Pearce EJ. Impairment of tetanus toxoid-specific Th1-like immune responses in humans infected with Schistosoma mansoni. J Infect Dis. 1996;173:269-72.

21. Nacher M. Interactions between worms and malaria: good worms or bad worms? Malar J. 2011:10:259.

22. Metenou S, Babu S, Nutman TB. Impact of filarial infections on coincident intracellular pathogens: Mycobacterium tuberculosis and Plasmodium falciparum. Curr Opin HIV AIDS. 2012;7:231-8.

23. Sokhna C, Hesran J-YL, Mbaye PA, Akiana J, Camara P, Diop M, et al. Increase of malaria attacks among children presenting concomitant infection by Schistosoma mansoni in Senegal. Malar J. 2004;3:43

24. Lyke KE, Dicko A, Dabo A, Sangare L, Kone A, Coulibaly D, et al. Association of Schistosoma haematobium infection with protection against acute Plasmodium falciparum malaria in Malian children. Am J Trop Med Hyg. 2005;73:1124-30.

25. Diallo TO, Remoue F, Schacht AM, Charrier N, Dompnier J-P, Pillet S, et al. Schistosomiasis co-infection in humans influences inflammatory markers in uncomplicated Plasmodium falciparum malaria. Parasite Immunol. 2004;26:365-9.

26. Hartgers FC, Obeng BB, Kruize YCM, Dijkhuis A, McCall M, Sauerwein RW, et al. Responses to malarial antigens are altered in helminth-infected children. J Infect Dis. 2009:199:1528-35.

27. Lyke KE, Dabo A, Sangare L, Arama C, Daou M, Diarra I, et al. Effects of concomitant Schistosoma haematobium infection on the serum cytokine levels elicited by acute Plasmodium falciparum malaria infection in Malian children. Infect Immun. 2006;74:5718-24

28. Lyke KE, Dabo A, Arama C, Daou M, Diarra I, Wang A, et al. Reduced T regulatory cell response during acute Plasmodium falciparum infection in Malian children co-infected with Schistosoma haematobium. PLoS One. 2012;7:e31647

29. Metenou S, Dembele B, Konate S, Dolo H, Coulibaly YI, Diallo AA, et al. Filarial infection suppresses malaria-specific multifunctional Th1 and Th17 responses in malaria and filarial coinfections. J Immunol. 2011;186:4725-33.
30. Adegnika AA, Zinsou JF, Issifou S, Ateba-Ngoa U, Kassa RF, Feugap EN, et al. Randomized, controlled, assessor-blind clinical trial to assess the efficacy of single- versus repeated-dose albendazole to treat Ascaris lumbricoides, Trichuris trichiura, and hookworm infection. Antimicrob Agents Chemother. 2014;58:2535-40

31. Ngoa UA, Zinsou JF, Kassa RFK, Feugap EN, Honkpehedji YJ, Massinga-Loembe $M$, et al. Assessment of the effect of Schistosoma haematobium co infection on malaria parasites and immune responses in rural populations in Gabon: study protocol. SpringerPlus. 2014;3:388.

32. Adegnika AA, Verweij JJ, Agnandji ST, Chai SK, Breitling LP, Ramharter M, et al. Microscopic and sub-microscopic Plasmodium falciparum infection, but not inflammation caused by infection, is associated with low birth weight. Am J Trop Med Hyg. 2006;75:798-803.

33. Genser B, Cooper PJ, Yazdanbakhsh M, Barreto ML, Rodrigues LC. A guide to modern statistical analysis of immunological data. BMC Immunol. 2007:8:27.

34. Helmy A, Antoniades CA, Guilfoyle MR, Carpenter KLH, Hutchinson PJ. Principal component analysis of the cytokine and chemokine response to human traumatic brain injury. PLoS One. 2012;7:e39677.

35. Everts B, Adegnika AA, Kruize YCM, Smits HH, Kremsner PG, Yazdanbakhsh M. Functional impairment of human myeloid dendritic cells during Schistosoma haematobium infection. PLoS Negl Trop Dis. 2010;4:e667.

36. Meurs L, Labuda L, Amoah AS, Mbow M, Ngoa UA, Boakye DA, et al. Enhanced pro-inflammatory cytokine responses following Toll-like-receptor ligation in Schistosoma haematobium-infected schoolchildren from rural Gabon. PLoS One. 2011;6:e24393.

37. Van der Kleij D, van den Biggelaar AHJ, Kruize YCM, Retra K, Fillie Y, Schmitz M, et al. Responses to Toll-like receptor ligands in children living in areas where schistosome infections are endemic. J Infect Dis. 2004;189:1044-51.

38. Van Riet E, Everts B, Retra K, Phylipsen M, van Hellemond JJ, Tielens AGM, et al. Combined TLR2 and TLR4 ligation in the context of bacterial or helminth extracts in human monocyte derived dendritic cells: molecular correlates for Th1/Th2 polarization. BMC Immunol. 2009;10:9.

39. Agrawal S, Agrawal A, Doughty B, Gerwitz A, Blenis J, Van Dyke T, et al. Cutting edge: different Toll-like receptor agonists instruct dendritic cells to induce distinct Th responses via differential modulation of extracellular signal-regulated kinase-mitogen-activated protein kinase and c-Fos. J Immunol. 2003;171:4984-9.

40. Wammes LJ, Wiria AE, Toenhake CG, Hamid F, Liu KY, Suryani H, et al. Asymptomatic plasmodial infection is associated with increased tumor necrosis factor receptor II-expressing regulatory T cells and suppressed type 2 immune responses. J Infect Dis. 2013;207:1590-9.

41. Turner JD, Meurs L, Dool P, Bourke CD, Mbow M, Dièye TN, et al. Schistosome infection is associated with enhanced whole-blood IL-10 secretion in response to cercarial excretory/secretory products. Parasite Immunol. 2013;35:147-56.

42. Smolen KK, Ruck CE, Fortuno 3rd ES, Ho K, Dimitriu P, Mohn WW, et al Pattern recognition receptor-mediated cytokine response in infants across 4 continents. J Allergy Clin Immunol. 2014;133:818-26.

43. Labuda LA, de Jong SE, Meurs L, Amoah AS, Mbow M, Ateba-Ngoa U, et al Differences in innate cytokine responses between European and African children. PLoS One. 2014;9:e95241.

44. Noone C, Parkinson M, Dowling DJ, Aldridge A, Kirwan P, Molloy SF, et al. Plasma cytokines, chemokines and cellular immune responses in pre-school Nigerian children infected with Plasmodium falciparum. Malar J. 2013;12:5.

45. Diallo TO, Remoue F, Gaayeb L, Schacht A-M, Charrier N, De Clerck D, et al. Schistosomiasis coinfection in children influences acquired immune response against Plasmodium falciparum malaria antigens. PLoS One. 2010;5:e12764

46. Netea MG, Quintin J, van der Meer JWM. Trained immunity: a memory for innate host defense. Cell Host Microbe. 2011:9:355-61. 